\title{
An Approximate Error Expression for RQAM Scheme under $\alpha-\eta-\mu$ Fading Conditions
}

\author{
N. KAPUCU
}

\begin{abstract}
In this paper, average symbol error rate (ASER) performance of rectangular quadrature amplitude modulation (RQAM) scheme is analyzed over $\alpha-\eta-\mu$ fading channels. First, an ASER expression is derived based on Chernoff approximation of Gaussian $Q$-function. Then, an asymptotic ASER formula is obtained for analyzing system behavior at high signal-to-noise ratio (SNR) regime. The ASER performance is presented for different modulation levels and fading parameter values. In addition, relative truncation error (RTE) is illustrated in order to determine how many terms are needed for the computation of proposed expression. It is shown that analytical results are in close agreement with exact results.
\end{abstract}

Index Terms- Error analysis, $\boldsymbol{\alpha}-\boldsymbol{\eta}-\boldsymbol{\mu}$ distribution, RQAM, Gaussian $Q$-function.

\section{INTRODUCTION}

$\mathrm{Q}$ UADRATURE amplitude modulation (QAM) which is known as efficient modulation method for bandwidth has an important role in digital multimedia transmission since it achieves high data rates. Rectangular QAM (RQAM), cross QAM (XQAM) and square QAM (SQAM) are popular QAM methods that are used in high speed communications. RQAM is considered as a generic modulation type and it has practical applications in the field of high speed mobile communications and microwave communications [1].

A number of studies which focus on the performance for RQAM schemes under different fading conditions, have been presented in the literature [2-7]. In [2], a lower bound ASER expression was derived for cooperative diversity systems with RQAM technique over Rayleigh fading channels. In addition, symbol error probability (SEP) and average symbol error rate (ASER) expressions were proposed for RQAM modulated systems under Nakagami- $m$ fading conditions [3-6]. In [3], the SEP of RQAM modulation was presented over Nakagami- $m$ fading channels in terms of the product of two Gaussian $Q$ functions. The authors in [4] studied the performance of $L$ branch communication system with RQAM scheme in the presence of Nakagami- $m$ fading and they proposed an

NURI KAPUCU, is with Department of Electrical and Electronics Engineering, Hitit University, Turkey, (e-mail:nurikapucu @hitit.edu.tr).

(iD https://orcid.org/0000-0002-8399-5546

Manuscript received September 28, 2018; accepted March 29, 2019. DOI: $10.17694 /$ bajece. 465158 expression for the SEP of the considered system. In [5] and [6], RQAM technique was applied to multiple relay networks and two-way relaying systems operating under Nakagami- $m$ fading conditions. Asghari et al. analyzed the SEP of RQAM scheme with maximum ratio combining over $\eta-\mu$ fading channels [7]. In [8], the ASER of RQAM and XQAM modulations were investigated based on moment generating function over two-wave with diffuse power fading channels. Lower bound ASER expressions of RQAM and XQAM for AF relaying systems were presented in Rayleigh fading with maximum ratio combining in [9]. In another work [10], the authors derived ASER formulas for hexagonal and rectangular QAM based on cumulative distribution function over Nakagami- $m$ fading channels.

In wireless communications, it is important to take the composite fading channels into consideration such as in [1115] for performance analysis since these fading models are generalized distributions which provide flexibility for reducing other well-known fading channels. However, as far as we know, error performance of RQAM modulated wireless communication systems over $\alpha-\eta-\mu$ fading channels does not exist in literature. $\alpha-\eta-\mu$ fading can be employed in order to reflect small variations in the signal strength and it has special cases including popular fading distributions such as Rayleigh, Nakagami- $m$, Weibull, $\eta-\mu, \alpha-\mu$. Motivated by this, for the first time in the literature, we analyze ASER performance of RQAM scheme over $\alpha-\eta-\mu$ fading channels. Here, we derive a novel ASER expression based on Chernoff approximation of Gaussian $Q$-function. Then, we also obtain an asymptotic ASER expression in order to evaluate the system behavior at high signal-to-noise ratio (SNR) region.

\section{SYSTEM AND CHANNEL MODELS}

We consider a single-input single-output wireless communication system that sends a signal $x$ which is modulated according to RQAM scheme. The received signal, $y$ is defined as

$$
y=x G+N_{0}
$$

where $G$ is the fading coefficient of the channel and $N_{0}$ is spectral density of noise power. The probability density function (PDF) of intantaneous SNR, $\gamma$, for $\alpha-\eta-\mu$ distribution is expressed by 


$$
\begin{gathered}
f_{\gamma}(\gamma)=A \exp \left(-\frac{2 \mu h \gamma^{\left(\frac{\alpha}{2}\right)}}{\bar{\gamma}^{\left(\frac{\alpha}{2}\right)}}\right) \gamma^{\left(\frac{\alpha(\mu+0.5)}{2}-1\right)} I_{\mu-\frac{1}{2}}\left(\frac{2 \mu H \gamma^{\left(\frac{\alpha}{2}\right)}}{\bar{\gamma}^{\left(\frac{\alpha}{2}\right)}}\right) \\
A=\left(\sqrt{\pi} \alpha \mu^{\mu+0.5} h^{\mu}\right) /\left(\Gamma(\mu) H^{\mu-0.5} \bar{\gamma}^{\lambda}\right)
\end{gathered}
$$

where $\alpha, \eta, \mu$ are fading parameters, $\lambda=\alpha(\mu+0.5) / 2$, $\Gamma(\cdot)$ is Gamma function, $I_{v}(\cdot)$ is the modified Bessel function of the first kind, $\bar{\gamma}$ is the average SNR defined by $\bar{\gamma}=E(\gamma)$ and $E(\cdot)$ denotes expectation. $h$ and $H$ parameters are defined in two different formats, respectively, as

$$
\begin{aligned}
& h=\frac{(1+\eta)^{2}}{4 \eta}, H=\frac{1-\eta^{2}}{4 \eta}, \quad 0<\eta<\infty, \text { Format } 1 \\
& h=\frac{1}{1-\eta^{2}}, H=\frac{\eta}{1-\eta^{2}}, \quad-1<\eta<1, \text { Format } 2
\end{aligned}
$$

\section{AVERAGE SYMBOL ERROR RATE ANALYSIS}

Mathematicaly, the ASER for any kind of modulation method is evaluated by integrating the conditional symbol error rate (SER) of additive White Gaussian noise (AWGN) channels over the PDF of instantaneous SNR as follows

$$
P_{s}(e)=\int_{0}^{\infty} P_{s}(e \mid \gamma) f_{\gamma}(\gamma) d \gamma
$$

where $P_{s}(e \mid \gamma)$ is the conditional SER expression of AWGN channels and $f_{\gamma}(\gamma)$ is the PDF of the instantaneous SNR. General order RQAM constellations can be obtained by combining two pulse amplitude modulation (PAM) signals as $M_{I}-P A M$ (in-phase) and $M_{Q}-P A M$ (quadrature). For $M$ ary RQAM, the conditional SER in AWGN channels is expressed as

$$
P_{s}(e \mid \gamma)=2(p Q(a \sqrt{\gamma})+q Q(b \sqrt{\gamma})-2 p q Q(a \sqrt{\gamma}) Q(b \sqrt{\gamma}))
$$

where $\quad M=M_{I} \times M_{Q}, \quad p=1-\left(1 / M_{I}\right)$, $a=\sqrt{6 /\left(\left(M_{I}^{2}-1\right)+\left(M_{Q}^{2}-1\right) \beta^{2}\right)}, \quad q=1-\left(1 / M_{Q}\right), \quad b=\beta a$, $\beta=d_{Q} / d_{I}$ is the decision distance ratio of quadrature-to-inphase components $\left(d_{I}\right.$ is the in-phase decision distance and $d_{Q}$ is the quadrature decision distance) and $Q(\cdot)$ is Gaussian $Q$-function. Inserting (6) into (5), the ASER can be rewritten as

$$
\begin{aligned}
P_{s}(e) & =\underbrace{\int_{0}^{\infty} 2 p Q(a \sqrt{\gamma}) f_{\gamma}(\gamma) d \gamma}_{I_{1}}+\underbrace{\int_{0}^{\infty} 2 q Q(b \sqrt{\gamma}) f_{\gamma}(\gamma) d \gamma}_{I_{2}} \\
& -\underbrace{\int_{0}^{\infty} 4 p q Q(a \sqrt{\gamma}) Q(b \sqrt{\gamma}) f_{\gamma}(\gamma) d \gamma}_{I_{3}}
\end{aligned}
$$

The integral in (7) is in an intractable format because of including Gaussian $Q$-function. Therefore, we utilize an upper bound approximation of the Gaussian $Q$-function which is defined by

$$
Q(x) \approx \frac{1}{2} \exp \left(-\frac{x^{2}}{2}\right)
$$

The approximate form given in (8) is known as Chernoff approximation [16]. This approximation facilitates the integration and ASER analysis over fading channels. First, we start by solving $I_{1}$ for ASER analysis. By inserting the Chernoff approximation and (2) into the first integral in (7), $I_{1}$ can be reexpressed as follows

$$
\begin{aligned}
I_{1}= & p A \int_{0}^{\infty} \exp \left(-\frac{a^{2} \gamma}{2}\right) \exp \left(-\frac{2 \mu h \gamma^{(\alpha / 2)}}{\bar{\gamma}^{(\alpha / 2)}}\right) \\
& \times \gamma^{\lambda-1} I_{\mu-0.5}\left(\frac{2 \mu H \gamma^{(\alpha / 2)}}{\bar{\gamma}^{(\alpha / 2)}}\right) d \gamma
\end{aligned}
$$

Now using the infinite series representations of exponential function $[17,(1.211 .1)]$ and $I_{v}(\cdot)[17,(8.445)]$ in (9), we have

$$
\begin{aligned}
I_{1} & =A p \sum_{k=0}^{\infty} \frac{(-1)^{k}}{k !}\left(\frac{2 \mu h}{\bar{\gamma}^{(\alpha / 2)}}\right)^{k} \sum_{m=0}^{\infty} \frac{(\mu H)^{u}}{m ! \Gamma(\mu+m+0.5)} \\
& \times \frac{1}{\left(\bar{\gamma}^{(\alpha / 2)}\right)^{u}} \int_{0}^{\infty} \exp \left(-\frac{a^{2} \gamma}{2}\right) \gamma^{v-1} d \gamma
\end{aligned}
$$

where $v=\lambda+(\alpha k / 2)+(\alpha u / 2)$ and $u=\mu+2 m-0.5$. After some algebra and by using $[17,(3.381 .4)], I_{1}$ is derived as

$$
I_{1}=A p \sum_{k=0}^{\infty} \frac{(-1)^{k}}{k !}\left(\frac{2 \mu h}{\bar{\gamma}^{(\alpha / 2)}}\right)^{k} \sum_{m=0}^{\infty} \frac{(\mu H)^{u} \Gamma(v)\left(a^{2} / 2\right)^{-v}}{m ! \Gamma(\mu+m+0.5)\left(\bar{\gamma}^{(\alpha / 2)}\right)^{u}}
$$

Then, $I_{2}$ and $I_{3}$ are obtained by using the same analytical steps as used for $I_{1}$, respectively, as 


$$
\begin{aligned}
I_{2} & =A q \sum_{k=0}^{\infty} \frac{(-1)^{k}}{k !}\left(\frac{2 \mu h}{\bar{\gamma}^{(\alpha / 2)}}\right)^{k} \sum_{m=0}^{\infty} \frac{(\mu H)^{u}}{\Gamma(\mu+m+0.5)} \\
& \times \frac{\Gamma(v)}{m !\left(\bar{\gamma}^{(\alpha / 2)}\right)^{u}\left(b^{2} / 2\right)^{v}} \\
I_{3} & =A p q \sum_{k=0}^{\infty} \frac{(-1)^{k}}{k !}\left(\frac{2 \mu h}{\bar{\gamma}^{(\alpha / 2)}}\right)^{k} \sum_{m=0}^{\infty} \frac{(\mu H)^{u}}{\Gamma(\mu+m+0.5)} \\
& \times \frac{\Gamma(v)}{m !\left(\bar{\gamma}^{(\alpha / 2)}\right)^{u}\left(\left(a^{2}+b^{2}\right) / 2\right)^{v}}
\end{aligned}
$$

Finally, the ASER expression is found as

$$
\begin{aligned}
P_{s}(e) & =A \sum_{k=0}^{\infty} \frac{(-1)^{k}}{k !}\left(\frac{2 \mu h}{\bar{\gamma}^{(\alpha / 2)}}\right)^{k} \sum_{m=0}^{\infty} \frac{(\mu H)^{u} \Gamma(v)}{m ! \Gamma(\mu+m+0.5)\left(\bar{\gamma}^{(\alpha / 2)}\right)^{u}} \\
& \times\left\{\frac{p}{\left(a^{2} / 2\right)^{v}}+\frac{q}{\left(b^{2} / 2\right)^{v}}-\frac{p q}{\left(\left(a^{2}+b^{2}\right) / 2\right)^{v}}\right\}
\end{aligned}
$$

Concerning about the truncation error which arises from the infinite series involved in (14), we evaluated the ASER expression in (14) for several values of the upper limits of infinite summations. Table I tabulates the ASER values and relative truncation error (RTE) for $4 \mathrm{X} 2 \mathrm{QAM}$ at $\bar{\gamma}=20 \mathrm{~dB}$ when $K$ and $M$ terms are used.

TABLE I

ASER AND RTE VALUES FOR 4X2 QAM WITH $\alpha=1.5, \eta=0.5, \mu=1.5$ AND $\beta=1$

\begin{tabular}{|c|c|c|c|}
\hline $\boldsymbol{M}$ & $\boldsymbol{K}$ & ASER & RTE \\
\hline 24 & 2 & $\mathbf{0 . 0 1 6 9 5 7 2 4 1 6 0 5 9 1 3}$ & $2.8012 \times 10^{-4}$ \\
\hline 24 & 3 & $\mathbf{0 . 0 1 6 9 5 7 2 9 5 8 3 5 4 0 5}$ & $3.1980 \times 10^{-6}$ \\
\hline 24 & 4 & $\mathbf{0 . 0 1 6 9 5 7 2 9 6 3 7 5 8 3 9}$ & $3.1870 \times 10^{-8}$ \\
\hline 24 & 5 & $\mathbf{0 . 0 1 6 9 5 7 2 9 6 3 8 0 7 0 1}$ & $2.8671 \times 10^{-10}$ \\
\hline 24 & 6 & $\mathbf{0 . 0 1 6 9 5 7 2 9 6 3 8 0 7 4 1}$ & $2.3755 \times 10^{-12}$ \\
\hline 24 & 7 & $\mathbf{0 . 0 1 6 9 5 7 2 9 6 3 8 0 7 4 2}$ & $1.8369 \times 10^{-14}$ \\
\hline 24 & 8 & $\mathbf{0 . 0 1 6 9 5 7 2 9 6 3 8 0 7 4 2}$ & $1.3382 \times 10^{-16}$ \\
\hline
\end{tabular}

RTE can be evaluated by following the same procedure in [18]. In Table I, one can see that the decimal places remained same even if the upper limits were increased. Hereby, Table I shows that the number of enough terms in order to compute the derived ASER expression in (14) with a negligible truncation error are $M=24$ and $K=8$. In Table II, we illustrate the ASER and RTE values for 8X4 QAM scheme at $\bar{\gamma}=40 \mathrm{~dB}$. Again, it can be easily seen that fewer terms are enough to evaluate the derived ASER expression at high SNR.
From Table II, only $K=4$ terms are needed for the evaluation of infinite series when $M=8$ where the RTE value decreases to $7.5473 \times 10^{-16}$. For all practical cases, (14) can be computed with a negligible truncation error without compromising numerical precision. Table I and Table II state that the derived expression is in rapidly convergent form.

TABLE II

ASER AND RTE VALUES FOR 8X4 QAM WITH $\alpha=1.5, \eta=0.5, \mu=1.5$ AND $\beta=1$

\begin{tabular}{|c|c|c|c|}
\hline$M$ & $\boldsymbol{K}$ & ASER & RTE \\
\hline 8 & 2 & $\mathbf{3 . 6 8 8 7 7 7 6 7 0 6 6 7 5 8 2 \times 1 0 ^ { - 5 }}$ & $4.5747 \times 10^{-8}$ \\
\hline 8 & 3 & $\mathbf{3 . 6 8 8 7 7 7 6 7 0 6 9 0 9 5 5} \times 10^{-5}$ & $6.3366 \times 10^{-12}$ \\
\hline 8 & 4 & $\mathbf{3 . 6 8 8 7 7 7 6 7 0 6 9 0 9 5 8} \times 10^{-5}$ & $7.5473 \times 10^{-16}$ \\
\hline 8 & 5 & $\mathbf{3 . 6 8 8 7 7 7 6 7 0 6 9 0 9 5 8} \times 10^{-5}$ & $8.0208 \times 10^{-20}$ \\
\hline
\end{tabular}

\section{ASYMPTOTIC ANALYSIS}

To analyze system behavior at the high SNR regime $(\bar{\gamma} \rightarrow \infty)$, we derive an asymptotic ASER expression for the considered system. Firstly, we expand the infinite series of exponential function and $I_{v}(\cdot)$ at zero point as

$$
\begin{gathered}
\exp \left(-2 \mu h \gamma^{(\alpha / 2)} / \bar{\gamma}^{(\alpha / 2)}\right) \approx 1 \\
I_{\mu-\frac{1}{2}}\left(\frac{2 H \mu \gamma^{(\alpha / 2)}}{\bar{\gamma}^{(\alpha / 2)}}\right) \approx \frac{\left(H \mu \gamma^{(\alpha / 2)} / \bar{\gamma}^{(\alpha / 2)}\right)^{\mu-0.5}}{\Gamma(\mu+0.5)}
\end{gathered}
$$

Thus, the asymptotic PDF expression becomes

$$
f_{\gamma}^{a}(\gamma)=\frac{A \gamma^{\left(\frac{\alpha(\mu+0.5)}{2}-1\right)\left(\frac{\alpha}{2}(\mu-0.5)\right)}}{\Gamma(\mu+0.5)}\left(\mu H \bar{\gamma}^{(-\alpha / 2)}\right)^{\mu-0.5}
$$

Then, substituting (17) and Chernoff approximation of Gaussian $Q$-function into (7), we have

$$
\begin{aligned}
& P_{s}^{a}(e)=\frac{A}{\Gamma(\mu+0.5)}\left(\frac{\mu H}{\bar{\gamma}^{(\alpha / 2)}}\right)^{\mu-0.5}\left\{p \int_{0}^{\infty} \exp \left(-\frac{\gamma a^{2}}{2}\right) \gamma^{\alpha \mu-1} d \gamma\right. \\
& \left.+q \int_{0}^{\infty} \exp \left(-\frac{\gamma b^{2}}{2}\right) \gamma^{\alpha \mu-1} d \gamma-p q \int_{0}^{\infty} \exp \left(-\frac{\gamma\left(a^{2}+b^{2}\right)}{2}\right) \gamma^{\alpha \mu-1} d \gamma\right\}
\end{aligned}
$$

By using [17, (3.381.4)], the asymptotic ASER expression results in 


$$
\begin{gathered}
P_{s}^{a}(e)=\frac{A \Gamma(\alpha \mu)}{\Gamma(\mu+0.5)}\left(\frac{\mu H}{\bar{\gamma}^{(\alpha / 2)}}\right)^{\mu-0.5}\left\{\frac{p}{\left(a^{2} / 2\right)^{\alpha \mu}}+\frac{q}{\left(b^{2} / 2\right)^{\alpha \mu}}\right. \\
\left.-\frac{p q}{\left(\left(a^{2}+b^{2}\right) / 2\right)^{\alpha \mu}}\right\}
\end{gathered}
$$

\section{RESULTS}

Here, ASER performance results are illustrated based on the proposed analytical expressions in comparison with exact results to validate the ASER expressions derived in this paper. The derived ASER expression includes convergent infinite series, which is truncated by $K$ and $M$ number of finite terms. Analytical results of (14) were obtained by setting the upper limits of the infinite summations to $K=10$ and $M=30$. Fig. 1 shows the ASER performance of $4 \mathrm{X} 2 \mathrm{QAM}$ scheme for several values of fading parameters. As can be seen from Fig. 1 , the analytical results of (14) match closely with the exact results while the asymptotic results are very tight at high SNR regime. A transition from $\alpha=1, \eta=0.5$ and $\mu=1.5$ to $\alpha=1.5, \quad \eta=0.3$ and $\mu=1.5$ provides a considerable performance improvement which is more than $5 \mathrm{~dB}$ even if the value of $\eta$ decreases from 0.5 to 0.3 with fixed value of $\mu$.

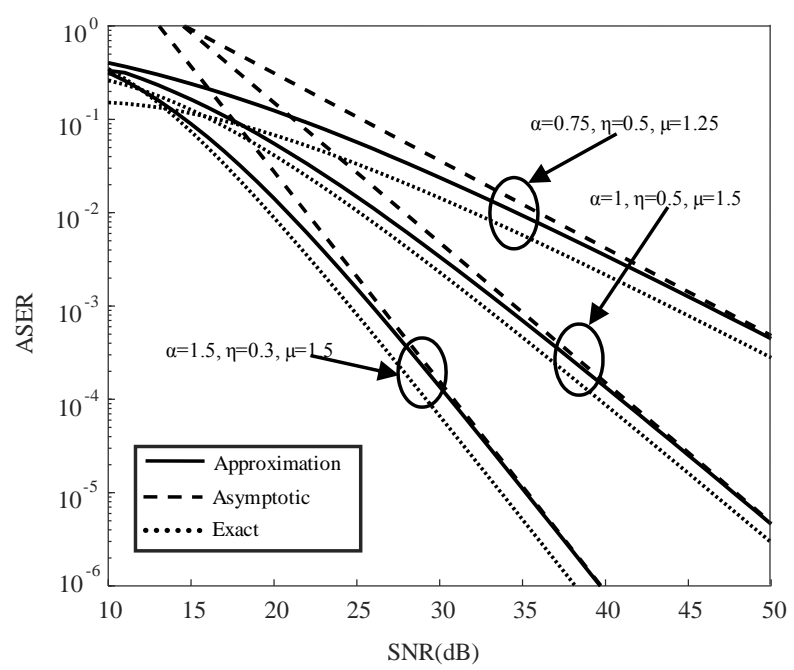

Fig.1. ASER performance of $4 \mathrm{X} 2 \mathrm{QAM}$ scheme with $\beta=1$

In Fig. 2, the ASER performance of 8X4 QAM modulated wireless systems is presented. Again, it can be seen that the exact results and approximated results are in close agreement. Moreover, the asymptotic results become tight with the approximate results at high SNR regime. For the case of $\alpha=1.5, \eta=0.3$ and $\mu=1.5,4 \mathrm{X} 2$ QAM scheme provides $P_{s}(e)=10^{-6}$ at $40 \mathrm{~dB}$ while the same ASER value is obtained at $46 \mathrm{~dB}$ with $8 \mathrm{X} 4 \mathrm{QAM}$ scheme. As expected, when the constellation size inceases, the performance decreases.

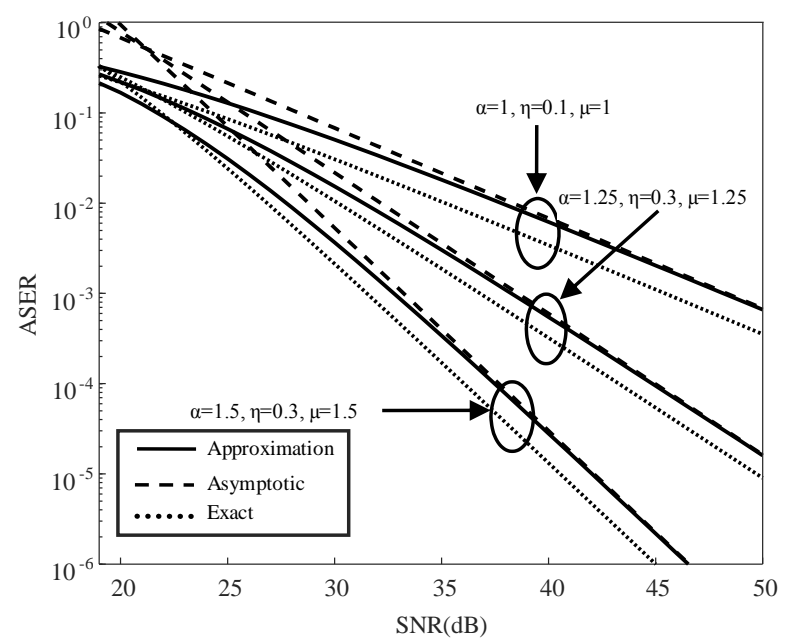

Fig.2. ASER performance of $8 \mathrm{X} 4 \mathrm{QAM}$ scheme with $\beta=1$.

\section{CONCLUSION}

We have derived approximated and asymptotic ASER expressions for wireless communication systems using RQAM scheme over $\alpha-\eta-\mu$ fading channels. The proposed approximate expression is in rapidly convergent form and its analytical results show close agreement to the exact ones. In addition, the asymptotic results are also tight with the approximate results at high SNR. In addition, it should be highlighted that using more terms for the infinite series does not have any influence in the 15th decimal place of the results and RTE values are decreasing rapidly to the negligible levels as given in Table I and Table II. As a result, one can easily obtain the ASER performance of the considered system over well-known fading channels such as Rayleigh, Nakagami- $m$, Weibull and so on by using the flexibility of $\alpha-\eta-\mu$ fading.

\section{REFERENCES}

[1] D. Dixit, , P.R. Sahu, "Performance analysis of rectangular QAM with $S C$ receiver over Nakagami- $m$ fading channels", IEEE Communications Letters, Vol.18, No.7, 2014, pp.1262-1265.

[2] D. Dixit, P. R. Sahu, "Symbol error rate of rectangular QAM with bestrelay selection in cooperative systems over Rayleigh fading channels", IEEE Communications Letters, Vol.16, No.4, 2012, pp.466-469.

[3] G. K. Karagiannidis, "On the symbol error probability of general order rectangular QAM in Nakagami- $m$ fading", IEEE Communications Letters, Vol.10, No.11, 2006, pp.745-747.

[4] X. Lei, P. Fan, L. Hao, "Exact symbol error probability of general order rectangular QAM with MRC diversity reception over Nakagami- $m$ fading channels", IEEE Communications Letters, Vol.11, No.12, 2007, pp.958-960.

[5] N. Kumar, P. K. Singya, V. Bhatia, "ASER analysis of hexagonal and rectangular QAM schemes in multiple-relay networks", IEEE Transactions on Vehicular Technology, Vol.67, No.2, 2018, pp.18151819.

[6] N. Kumar, V. Bhatia, "Exact ASER analysis of rectangular QAM in two-way relaying networks over Nakagami- $m$ fading channels", IEEE Wireless Communications Letters, Vol.5, No.5, 2016, pp.548-551.

[7] V. Asghari, D.B. da Costa, S. Aissa, "Symbol error probability of rectangular QAM in MRC systems with correlated $\eta-\mu$ fading channels", IEEE Transactions on Vehicular Technology, Vol.59, No.3, 2010, pp.1497-1503. 
[8] D. Dixit, P.R. Sahu, "Performance of QAM signaling over TWDP fading channels", IEEE Transactions on Wireless Communications, Vol.12, No.4, 2013, pp.1794-1799.

[9] N. Kumar, V. Bhatia, D. Dixit, "Performance analysis of QAM in amplify-and-forward cooperative communication networks over Rayleigh fading channels", AEU-International Journal of Electronics and Communications, Vol.72, 2017, pp.86-94.

[10] P. R. Singya, N. Kumar, V. Bhatia, "Impact of imperfect CSI on ASER of hexagonal and rectangular QAM for AF relaying network", IEEE Communications Letters, Vol.22, No.2, 2018, pp.428-431.

[11] N. Kapucu, M. Bilim, I. Develi, "Outage performance of cooperative DS-CDMA systems with best path selection over $\alpha-\eta-\mu$ fading channels", Electronics Letters, Vol.53, No.11, 2017, pp.752-754.

[12] O.S. Badarneh, M.S. Aloqlah, "Performance analysis of digital communication systems over $\alpha-\eta-\mu$ fading channels", IEEE Transactions on Vehicular Technology, Vol.65, No.10, 2016, pp.79727981.

[13] J. M. Moualeu, D. B. da Costa, W. Hamouda, U. S. Dias, R. A. A. de Souza, "Physical layer security over $\alpha-\kappa-\mu$ and $\alpha-\eta-\mu$ fading channels", IEEE Transactions on Vehicular Technology, Vol.68, No.1, 2019, pp. $1025-1029$.

[14] A. M. Magableh, T. Aldalgamouni, S. Mater, O. S. Badarneh, "Ergodic capacity and SNR analysis for dual hop amplify and forward cooperative communication systems over $\alpha-\eta-\mu$ channels", IEEE Transactions on Vehicular Technology, Vol.67, No.12, 2018, pp.12384 $-12388$.

[15] N. Kapucu, M. Bilim, "Analysis of analytical capacity for FisherSnedecor $F$ fading channels with different transmission schemes", Electronics Letters, Vol.55, No.5, 2019, pp.283-285.

[16] M.K. Simon, M.-S. Alouini, Digital Communication over Fading Channels, John Wiley \& Sons, 2000.

[17] I.S. Gradshteyn, I.M. Ryzhik, Table of Integrals, Series and Products, Academic Press, 2000.

[18] S.S. Soliman, N.C. Beaulieu, "The bottleneck effect of Rician fading in dissimilar dual-hop AF relaying systems", IEEE Transactions on Vehicular Technology, Vol.63, No.4, 2014, pp.1957-1965.

\section{BIOGRAPHIES}

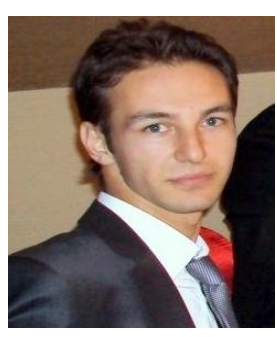

Nuri Kapucu received the B.Sc., M.Sc. and Ph.D. degrees in Electrical and Electronics Engineering from Erciyes University, Turkey, in 2010, 2012 and 2017, respectively. From 2011 to 2018, he was a Research Assistant in the department of Electrical and Electronics Engineering at Erciyes University. Currently, he is an assistant professor in the department of Electrical and Electronics Engineering at Hitit University. His current research interests include performance analysis over fading channels, cooperative communications, two-way relaying, and multiple access techniques. 